\title{
2-P-139 Poster Sessions
}

\section{A dendritic spine shrinkage in response to extracellular high $\mathrm{K}^{+}$is inhibited by $\mathrm{Co}^{2+}$ or $\mathrm{Cd}^{2+}$}

\author{
Naoko Konishi, Toshinori Sawano, Jin Nakatani, Hidekazu Tanaka
}

Lab. Pharrm., Grad.sch. Life sci., Ritsumeikan Univ.

In the brain, neurons communicate with each other at excitatory and inhibitory synapses. In each excitatory synapse, a dendritic spine receives the excitatory neurotransmitter released by an axon terminal. An extracellular high $\mathrm{K}^{+}$induces robust depolarization and mimics massive synaptic transmission. A dendritic spine transiently shrinks in high $\mathrm{K}^{+}$ solution. In this study, we investigated the mechanisms of the spine shrinkage using cultured hippocampal neurons. The spine shrinkage observed in response to high $\mathrm{K}^{+}$was inhibited in the presence of extracellular $\mathrm{Co}^{2+}$ or $\mathrm{Cd}^{2+}$. Then we investigated $\mathrm{Ca}^{2+}$-signaling pathway because these metal ions are supposed to be wide spectrum $\mathrm{Ca}^{2+}$-channel blockers. We preincubated neurons with ROCK inhibitor Y27632 or CaMKII inhibitor KN-93. However, neither of them inhibited the spine shrinkage. Finally, we tested whether myosin is involved in the spine shrinkage. A myosin ATPase inhibitor, blebbistatin, did not inhibit the spine shrinkage in high $\mathrm{K}^{+}$. Taken together, extracellular high $\mathrm{K}+-$ triggered $\mathrm{Ca}^{2+}$-influx seem to induce the spine shrinkage; however, the downstream signaling pathway or the motor machinery has not yet been determined. 